\title{
Thalamotomy-Like Effects From Partial Removal of a Ventral Intermediate Nucleus Deep Brain Stimulator Lead in a Patient With Essential Tremor: Case Report
}

\author{
John D. Rolston, MD, PhD*, Alexander D. Ramos, $\mathrm{PhD}^{\star}$, Susan Heath, MSN, RN ${ }^{\ddagger}$, Dario J. \\ Englot, MD, $\mathrm{PhD}^{*}$, and Daniel A. Lim, MD, $\mathrm{PhD}^{*}, \S$ \\ *Department of Neurological Surgery, University of California, San Francisco, California \\ ¥San Francisco VA Parkinson's Disease Research, Education, \& Clinical Center, San Francisco, \\ California \\ §Surgical Service, San Francisco Veterans Affairs Medical Center, San Francisco, California
}

\begin{abstract}
BACKGROUND AND IMPORTANCE-The ventral intermediate nucleus of the thalamus is a primary target of deep brain stimulation (DBS) in patients with essential tremor. Despite reliable control of contralateral tremor, there is sometimes a need for lead revision in cases of infection, hardware malfunction, or failure to relieve symptoms. Here, we present the case of a patient undergoing revision after ventral intermediate nucleus (Vim) DBS failed to control his tremor. During the electrode removal, the distal portion of the lead was found to be tightly adherent to tissue within the deep brain. Partial removal of the electrode in turn caused weakness, paresthesias, and tremor control similar to the effects produced by thalamotomy or thalamic injury.
\end{abstract}

CLINICAL PRESENTATION-A 48-year-old man with essential tremor had bilateral Vim DBS leads implanted 10 years earlier but had poor control of his tremor and ultimately opted for surgical revision with lead placement in the zona incerta. During attempted removal of his right lead, the patient became somnolent with contralateral weakness and paresthesias. The procedure was aborted, and postoperative neuroimaging was immediately obtained, showing no signs of stroke or hemorrhage. The patient had almost complete control of his left arm tremor postoperatively, and his weakness soon resolved.

CONCLUSION-To the best of our knowledge, this is the first reported case of cerebral injury after DBS revision and offers insights into the mechanism of high-frequency electric stimulation compared with lesions. That is, although high-frequency stimulation failed to control this patient's tremor, thalamotomy-like injury was completely effective.

Correspondence: Daniel A. Lim, MD, PhD, 35 Medical Center Way, RMB 1037, San Francisco, CA 94143-0525. daniel.lim@ucsf.edu.

Disclosures

The other authors have no personal, financial, or institutional interest in any of the drugs, materials, or devices described in this article. 


\section{Keywords}

Deep brain stimulation; Essential tremor; Thalamotomy; Ventral intermediate nucleus; Zona incerta

Essential tremor is a common and sometimes debilitating movement disorder affecting up to $5 \%$ of patients $>60$ years of age. ${ }^{1}$ The action and postural tremors these patients experience most often affect the arms and hands but can also affect the head, legs, voice, and trunk. Medications such as propranolol and primidone control symptoms in many patients, but about $50 \%$ of patients remain refractory to medical therapy. ${ }^{1}$

For medically refractory essential tremor patients, deep brain stimulation (DBS) of the thalamic ventral intermediate nucleus (Vim) is an accepted alternative treatment ${ }^{2-5}$ although it is not universally effective and sometimes requires lead revision in cases of infection, hardware malfunction, or failure to relieve symptoms. Failures in symptom control, in particular, have been reported to range from $6.9 \%$ to $18.5 \%$ of treated patients. ${ }^{6,7} \mathrm{An}$ alternative to thalamic stimulation is thalamotomy, in which a permanent lesion is created at the Vim. Although this procedure can be as efficacious as DBS in reducing clinical symptoms, it is associated with higher rates of adverse events and is irreversible, and for this reason, DBS lead placement is the recommended surgical treatment for most cases of essential tremor. ${ }^{8-10}$

Several studies have examined alternatives to Vim stimulation for those patients who do not benefit from that target such as subthalamic nucleus stimulation ${ }^{11,12}$ and directional current steering. ${ }^{13}$ One of the most promising alternatives is perhaps stimulation of the caudal zona incerta (ZI). ${ }^{11,14}$ The ZI target appears to offer tremor control similar to Vim stimulation but at reduced voltages, which limits side effects such as disequilibrium and dysarthria. ${ }^{15}$ Moreover, a study by Blomstedt et al ${ }^{16}$ showed beneficial tremor control in patients who had failed standard Vim stimulation and subsequently underwent ZI electrode implantation.

Here, we present a patient who initially underwent bilateral Vim DBS electrode implantation for essential tremor. However, this stimulation was ineffective at alleviating his symptoms, prompting an attempt to retarget the patient's leads to the bilateral ZI. Details of the case and outcome follow.

\section{CLINICAL PRESENTATION}

This 48-year-old male patient was diagnosed with essential tremor in his 20s, and the tremor progressed over time. After failing medical management, he had left and then right Vim DBS electrodes placed 13 and 12 years, respectively, before the procedure described here. Although he had partial tremor relief of his right hand (left-sided system), the right-sided system (left hand) was never as successful, despite DBS lead locations within $1.2 \mathrm{~mm}$ (Table 1) of locations known to provide tremor control. ${ }^{17}$ As the patient's tremor worsened, both systems became even less useful. The patient was not followed up by our practice during this initial period, so we do not have quantitative data assessing his initial tremor control. Parameters used by the patient are presented in Table 2. The patient continued to worsen so 
that, before the present surgery, the patient was unable to write, pour water, tie his shoes, or work with the stimulator on. With or without stimulation, his left arm tremor was $4 / 4$ on the Fahn-Tolosa-Marín scale.

Because the system was not relieving the patient's tremor, we discussed a possible revision from bilateral Vim to ZI targets. The risks, benefits, and alternatives of the procedure were discussed with the patient, who requested that we proceed. The patient additionally consented to the use of his information for clinical studies and case reports as part of Institutional Review Board- approved protocol 10-02-130.

Because of the particulars of this patient's anatomy and the location of the existing VIM DBS leads, we were unable to develop surgical trajectories that did not cross the previously implanted electrodes, so it was determined that they must be removed. The right-sided system was targeted first because it offered the least benefit to the patient. An incision was made over the right-sided scar using sharp dissection and monopolar cautery. Care was taken not to expose the electrode or wiring to monopolar current. The Medtronic StimLoc cap was readily identified and easily removed. The anchoring clip was also easily removed. Scar tissue around the electrode entry site was mobilized with the use of curettes and a Penfield number 4 .

Once the electrode was freely mobile at its cranial insertion point and its entry point in the cortex was visualized, we attempted to remove it from the brain. The first $1 \mathrm{~cm}$ offered moderate resistance. However, further attempts at electrode removal were met with increasing resistance. To investigate the source of this resistance, we used a Medtronic Oarm for lateral fluoroscopy (Figure 1). With live fluoroscopic imaging, we noted that the electrode appeared to "spring" back to a deeper location after withdrawal tension was removed from the lead (this dynamic action of the lead was not captured with the still images obtained during fluoroscopy). Because of this evidence suggesting physical resistance to lead removal, we did not continue with further attempts to remove the lead.

Throughout the operation, we maintained conversation with the patient to monitor his neurological status. Shortly after we encountered resistance to lead removal, the patient became increasingly somnolent. We immediately ceased manipulation of the electrode and stopped all sedation. The patient gradually became more alert over the next 4 to 5 minutes, after which he was alert and fully oriented. On complete neurological examination, however, we noted a new left facial droop, $3 / 6$ on the House-Brackmann scale. The rest of his neurological examination remained intact, with essentially full strength in his left hand, arm, and leg, in particular. Finger-to-nose movements were enacted without difficulty. Most important, the patient had no notable tremor (0/4 on the Fahn-Tolosa-Marín scale) in his left hand or arm, a tremendous improvement from his preoperative baseline. Given the improvement in tremor, the change in examination, and difficulty removing the electrode, the remainder of the procedure was aborted.

The immediate postoperative head computed tomography did not show any signs of hemorrhage or stroke (Figure 2), and the patient was taken to the intensive care unit for close monitoring. His neurological examination showed continued slight left facial droop ( $3 / 6$ on 
the House-Brackmann scale); $4+/ 5$ strength in his left biceps, triceps, and grip; and numbness in the left perioral area and in the first 3 digits of his left hand. The remainder of his examination was normal. His tremor remained completely controlled in his left arm and hand.

The next morning (postoperative day 1), the patient's examination improved such that he had full $5 / 5$ strength in his left arm and hand. His finger numbness spread to include the entire left hand. His facial droop improved to $2 / 6$ on the House-Brackmann scale. A magnetic resonance imaging scan obtained approximately 24 hours after the operation revealed T2 hyperintensity at the tip of the right DBS lead in the region of the Vim (Figure $3 \mathrm{~A}$ and $3 \mathrm{~B}$ ). Although there was increased diffusion-weighted imaging signal in this region (Figure 3C), there was no corresponding diffusion restriction on the apparent diffusion coefficient map (Figure 3D), making the diffusion-weighted imaging signal more likely to be T2 shine-through. Taken together, these findings suggest local edema without evidence of stroke or hemorrhage.

The patient underwent physical and occupational therapy evaluation while admitted and was discharged home on his second postoperative day. At the 2.5-month follow-up, the patient's tremor remained well controlled with only occasional "break-through" tremor during some actions, per the patient. At this time, he had persistent paresthesias in the left side of his mouth, tongue, and first 2 digits of his left hand. He also complained of slight dysarthria and left leg weakness with sustained, strenuous activity, along with depressed mood.

At the 6-month follow-up, the patient's paresthesias and slight dysarthria persisted, but his strength improved. The patient had no arm or leg resting or postural tremor $(0 / 4$ on the FahnTolosa-Marín scale) but had slight intention tremor on finger-nose-finger testing ( $1 / 4$ on the Fahn-Tolosa-Marín scale). He had mild left facial weakness (2/6 on the House-Brackmann) and mild left biceps weakness (4+/5) but was full strength (5/5) in all other tested muscle groups. His intermittent gait and leg weakness were completely resolved. Magnetic resonance imaging at this time showed a cystic space in the location of the partially removed lead (Figure 4). The lead was never seen to cross a ventricle on any imaging.

\section{DISCUSSION}

Complications of DBS surgery are well documented. Symptomatic hemorrhage occurs in $1.1 \%$ to $1.4 \%$, wound infection in $1.7 \%$ to $8 \%$, and hardware failure (eg, fracture, malfunction) in $1.5 \%$ to $36 \%$, in addition to rarer complications like seizures, hypotension, and ischemic stroke. ${ }^{18-21}$ To the best of our knowledge, complications induced by hardware removal such as that experienced by our patient have not been reported.

We hypothesize that the particular complication experienced by our patient was caused by adherence of the distal portion of the electrode to the deep brain parenchyma. Because the electrode remained implanted, we were not able to directly assess the lead to gain better insight into the source of the resistance to its removal. However, given the location of the postoperative edema, we believe that it was likely most adherent to tissues at the distal tip at 
or near the electrode contacts. There was no evidence of edema or injury along the more proximal electrode, suggesting that the adherence was mostly distal.

The degree of the induced lesion is best seen on the 6-month follow-up magnetic resonance imaging, which shows a cystic area in the location of the partially removed right lead (Figure 4). Given this imaging, it is likely that the area around the tip of the electrode was permanently injured, and we expect the tremor control to persist as a result of this lesion. Consistent with this, the patient's tremor remained controlled at both the 2.5- and 6-month follow-up.

Prior studies have examined the histological changes induced by DBS electrodes. ${ }^{22}$ Most electrodes, although not all, are surrounded by a 5- to $25-\mu \mathrm{m}$ fibrous sheath across the entire length, which is thickest at actively stimulating electrodes and directly related to charge density. ${ }^{23}$ There is also frequently an inflammatory component consisting of giant cells and macro-phages, which sometimes contains trace amounts of metallic inclusions, presumed to be platinum or iridium shed from the electrode contacts. ${ }^{24}$ Again, we are not able to examine our patient's electrode or tissue to determine what degree of encapsulation was present. It is possible, however, that a fibrous sheath existed, which was somehow intercalated with the electrode contacts, causing distal strain when removal was attempted. The electrode contacts of DBS leads are metallic cylinders with an exposed junction between the insulating material and contact, which might form a natural space for adhesions to form.

The mechanism of high-frequency electric stimulation in tremor relief is an area of active research. ${ }^{25}$ On the basis of the similar effects of stimulation and stereotactic lesions, the mechanism was initially believed to be inhibition of neurons, possibly by stimulating local inhibitory synapses. ${ }^{26}$ Later research using electric recording, ${ }^{27,28}$ positron emission tomography, ${ }^{29,30}$ and functional magnetic resonance imaging ${ }^{31}$ showed that stimulated sites appeared, in fact, to be highly activated by stimulation rather than inhibited. How this corresponds to the similar effects of lesioning remains unknown, although the effect is perhaps due to an "informational lesion" in which the pattern of evoked activity is somehow functionally equivalent to the absence of activity created via lesioning. ${ }^{32}$

The lesion created in our patient from attempted electrode removal led to an immediate and sustained improvement in contralateral tremor control, yet stimulation at the same site had no such effect. This apparent dissociation of therapeutic effects provides some information about the mechanism of stimulation as it relates to lesioning. As the many functional studies cited above have shown, stimulation is not physiologically equivalent to lesioning. In contrast to the relatively complete inactivation caused by lesioning, it is thought that stimulation results in elevated, synchronous activation of neural ensembles. In our patient, this putative synchronous activity failed to effectively treat his tremor, yet the patient's postoperative course suggests that the ablation of local neural activity can still be effective. An implication of this, especially as electrode designs and stimulation protocols become more nuanced and elaborate, is that stimulation designed to actively inhibit Vim output, rather than synchronize output at high frequencies, holds potential as an alternative means of relieving tremor. ${ }^{33-35}$ If direct inhibition of Vim by electric stimulation is not possible, alternatives might be targeting upstream nuclei of Vim that would, in turn, lead to its 
inhibition or possibly targeting downstream nuclei of Vim to alter their activity directly. 36 Finally, our case report suggests that using existing Vim electrodes for thalamotomies by means of radiofrequency lesioning may be a viable alternative approach to achieving tremor control. ${ }^{37}$ At the same time, the mechanism of lesioning in the present report is very different from that of radiofrequency lesioning. Therefore, any connection, although interesting, is speculative.

The initial plan for our patient was to revise his electrodes from Vim to ZI, which has shown some success in rescuing tremor control in patients in whom Vim stimulation has failed. ${ }^{16}$ Because of the close proximity between Vim and ZI, it is frequently the case that the 2 electrode trajectories cross and that the Vim electrode must be removed before the ZI electrode is inserted, as in our case. There are occasions, however, when the prior electrode can remain in place, as in 1 of the 5 patients reported by Blomstedt et al ${ }^{16}$ during their Vimto-ZI revision surgeries. Leaving the prior electrode in place would have prevented the complication we observed in our patient but unfortunately was not possible given trajectory considerations. Moving forward, another alternative would be to plan trajectories that incorporate both the Vim and ZI to maximize the stimulation sites and to make conversion between areas a matter of reprogramming rather than reoperation. This has been shown feasible in at least 1 case series by Chang et $\mathrm{al}^{38}$ in which both locations were targeted bilaterally in 5 patients with good effect.

\title{
CONCLUSION
}

Although complications of DBS surgery have been documented, this is, to the best of our knowledge, the first reported case of cerebral injury after attempted lead removal. After the attempted revision, our patient has almost complete control of his tremor, whereas previously, the implanted DBS lead offered poor tremor control. Therefore, this case, offers the unique opportunity to compare the efficacy of a thalamotomy-like lesion with DBS at the same anatomic site in the same patient. The outcome of this case demonstrates that lesioning is not always functionally equivalent to DBS and suggests that lesioning can be effective for tremors refractory to DBS treatment. Furthermore, this case provides insight into the potential mechanism of action of stimulation compared with lesions and suggests new programming strategies designed to actively inhibit thalamic targets rather than to stimulate them.

\section{Acknowledgments}

Research activities of Dr Lim are supported by grants from the California Institute for Regenerative Medicine (CIRM RT2-01975), the Veterans Affairs (1I01 BX000252-04), the Shurl and Kay Curci Foundation, and the Sontag Foundation. Dr Lim is a consultant to Accurexa, Inc. S. Heath is a consultant and educator for Medtronic and has a relative who is employed by Medtronic.

\section{ABBREVIATIONS}

\author{
DBS deep brain stimulation \\ Vim ventral intermediate nucleus
}




\section{REFERENCES}

1. Lyons KE, Pahwa R. Deep brain stimulation and essential tremor. J Clin Neurophysiol. 2004; 21(1): 2-5. [PubMed: 15097289]

2. Rehncrona S, Johnels B, Widner H, Tornqvist AL, Hariz M, Sydow O. Long-term efficacy of thalamic deep brain stimulation for tremor: double-blind assessments. Mov Disord. 2003; 18(2): 163-170. [PubMed: 12539209]

3. Hariz MI, Krack P, Alesch F, et al. Multicentre European study of thalamic stimulation for parkinsonian tremor: a 6 year follow-up. J Neurol Neurosurg Psychiatry. 2008; 79(6):694-699. [PubMed: 17898034]

4. Sydow O, Thobois S, Alesch F, Speelman JD. Multicentre European study of thalamic stimulation in essential tremor: a six year follow up. J Neurol Neurosurg Psychiatry. 2003; 74(10):1387-1391. [PubMed: 14570831]

5. Pahwa R, Lyons KL, Wilkinson SB, et al. Bilateral thalamic stimulation for the treatment of essential tremor. Neurol. 1999; 53(7):1447-1450.

6. Benabid AL, Pollak P, Gao D, et al. Chronic electrical stimulation of the ventralis intermedius nucleus of the thalamus as a treatment of movement disorders. J Neurosurg. 1996; 84(2):203-214. [PubMed: 8592222]

7. Koller W, Pahwa R, Busenbark K, et al. High-frequency unilateral thalamic stimulation in the treatment of essential and parkinsonian tremor. Ann Neurol. 1997; 42(3):292-299. [PubMed: 9307249]

8. Pahwa R, Lyons KE, Wilkinson SB, et al. Comparison of thalamotomy to deep brain stimulation of the thalamus in essential tremor. Mov Disord. 2001; 16(1):140-143. [PubMed: 11215575]

9. Schuurman PR, Bosch DA, Bossuyt PM, et al. A comparison of continuous thalamic stimulation and thalamotomy for suppression of severe tremor. N Engl J Med. 2000; 342(7):461-468. [PubMed: 10675426]

10. Tasker RR. Deep brain stimulation is preferable to thalamotomy for tremor suppression. Surg Neurol. 1998; 49(2):145-153. discussion 153-154. [PubMed: 9457264]

11. Blomstedt P, Sandvik U, Linder J, Fredricks A, Forsgren L, Hariz MI. Deep brain stimulation of the subthalamic nucleus versus the zona incerta in the treatment of essential tremor. Acta Neurochirurgica. 2011; 153(12):2329-2335. [PubMed: 21904970]

12. Lind G, Schechtmann G, Lind C, Winter J, Meyerson BA, Linderoth B. Subthalamic stimulation for essential tremor: short- and long-term results and critical target area. Stereotact Funct Neurosurg. 2008; 86(4):253-258. [PubMed: 18552522]

13. Keane M, Deyo S, Abosch A, Bajwa JA, Johnson MD. Improved spatial targeting with directionally segmented deep brain stimulation leads for treating essential tremor. J Neural Eng. 2012; 9(4):046005. [PubMed: 22732947]

14. Plaha P, Khan S, Gill SS. Bilateral stimulation of the caudal zona incerta nucleus for tremor control. J Neurol Neurosurg Psychiatry. 2008; 79(5):504-513. [PubMed: 18037630]

15. Lozano AM, Levy R. Reoperation of deep brain stimulation in patients with essential tremor. World Neurosurg. 2012; 78(5):442-444. [PubMed: 22373891]

16. Blomstedt P, Lindvall P, Linder J, Olivecrona M, Forsgren L, Hariz MI. Reoperation after failed deep brain stimulation for essential tremor. World Neurosurg. 2012; 78(5):554.e551-554.e555. [PubMed: 22381301]

17. Papavassiliou E, Rau G, Heath S, et al. Thalamic deep brain stimulation for essential tremor: relation of lead location to outcome. Neurosurgery. 2008; 62(suppl 2):884-894. [PubMed: 18596419]

18. Fenoy AJ, Simpson RK Jr. Risks of common complications in deep brain stimulation surgery: management and avoidance. J Neurosurg. 2014; 120(1):132-139. [PubMed: 24236657] 
19. Sillay KA, Larson PS, Starr PA. Deep brain stimulator hardware-related infections: incidence and management in a large series. Neurosurgery. 2008; 62(2):360-366. discussion 366-367. [PubMed: 18382313]

20. Binder DK, Rau G, Starr PA. Hemorrhagic complications of microelectrode-guided deep brain stimulation. Stereotact Funct Neurosurg. 2003; 80(1-4):28-31. [PubMed: 14745205]

21. Tong F, Ramirez-Zamora A, Gee L, Pilitsis J. Unusual complications of deep brain stimulation. Neurosurg Rev. 2015; 38(2):245-252. discussion 252. [PubMed: 25342239]

22. van Kuyck K, Welkenhuysen M, Arckens L, Sciot R, Nuttin B. Histological alterations induced by electrode implantation and electrical stimulation in the human brain: a review. Neuromodulation. 2007; 10(3):244-261. [PubMed: 22150838]

23. Haberler C, Alesch F, Mazal PR, et al. No tissue damage by chronic deep brain stimulation in Parkinson's disease. Ann Neurol. 2000; 48(3):372-376. [PubMed: 10976644]

24. Moss J, Ryder T, Aziz TZ, Graeber MB, Bain PG. Electron microscopy of tissue adherent to explanted electrodes in dystonia and Parkinson's disease. Brain. 2004; 127:2755-2763. [PubMed: 15329356]

25. Miocinovic S, Somayajula S, Chitnis S, Vitek JL. History, applications, and mechanisms of deep brain stimulation. JAMA Neurol. 2013; 70(2):163-171. [PubMed: 23407652]

26. Benazzouz A, Gao DM, Ni ZG, Piallat B, Bouali-Benazzouz R, Benabid AL. Effect of highfrequency stimulation of the subthalamic nucleus on the neuronal activities of the substantia nigra pars reticulata and ventrolateral nucleus of the thalamus in the rat. Neuroscience. 2000; 99(2):289295. [PubMed: 10938434]

27. Hashimoto T, Elder CM, Okun MS, Patrick SK, Vitek JL. Stimulation of the subthalamic nucleus changes the firing pattern of pallidal neurons. J Neurosci. 2003; 23(5):1916-1923. [PubMed: 12629196]

28. Anderson ME, Postupna N, Ruffo M. Effects of high-frequency stimulation in the internal globus pallidus on the activity of thalamic neurons in the awake monkey. J Neurophysiol. 2003; 89(2): 1150-1160. [PubMed: 12574488]

29. Hershey T, Revilla FJ, Wernle AR, et al. Cortical and subcortical blood flow effects of subthalamic nucleus stimulation in PD. Neurology. 2003; 61(6):816-821. [PubMed: 14504327]

30. Perlmutter JS, Mink JW, Bastian AJ, et al. Blood flow responses to deep brain stimulation of thalamus. Neurology. 2002; 58(9):1388-1394. [PubMed: 12011286]

31. Jech R, Urgosik D, Tintera J, et al. Functional magnetic resonance imaging during deep brain stimulation: a pilot study in four patients with Parkinson's disease. Mov Disord. 2001; 16(6): 1126-1132. [PubMed: 11748747]

32. Grill WM, Snyder AN, Miocinovic S. Deep brain stimulation creates an informational lesion of the stimulated nucleus. Neuroreport. 2004; 15(7):1137-1140. [PubMed: 15129161]

33. Reato D, Rahman A, Bikson M, Parra LC. Low-intensity electrical stimulation affects network dynamics by modulating population rate and spike timing. J Neurosci. 2010; 30(45):15067-15079. [PubMed: 21068312]

34. Bikson M, Lian J, Hahn PJ, Stacey WC, Sciortino C, Durand DM. Suppression of epileptiform activity by high frequency sinusoidal fields in rat hippocampal slices. J Physiol. 2001; 531(pt 1): 181-191. [PubMed: 11179402]

35. Hariz M. Deep brain stimulation: new techniques. Parkinsonism Relat Disord. 2014; 20(suppl 1):S192-S196. [PubMed: 24262179]

36. Klein JC, Barbe MT, Seifried C, et al. The tremor network targeted by successful VIM deep brain stimulation in humans. Neurology. 2012; 78(11):787-795. [PubMed: 22377809]

37. Strickland BA, Jimenez-Shahed J, Jankovic J, Viswanathan A. Radiofrequency lesioning through deep brain stimulation electrodes: a pilot study of lesion geometry and temperature characteristics. J Clin Neurosci. 2013; 20(12):1709-1712. [PubMed: 24035651]

38. Chang WS, Chung JC, Kim JP, Chang JW. Simultaneous thalamic and posterior subthalamic electrode insertion with single deep brain stimulation electrode for essential tremor. Neuromodulation. 2013; 16(3):236-243. discussion 243. [PubMed: 22985104] 


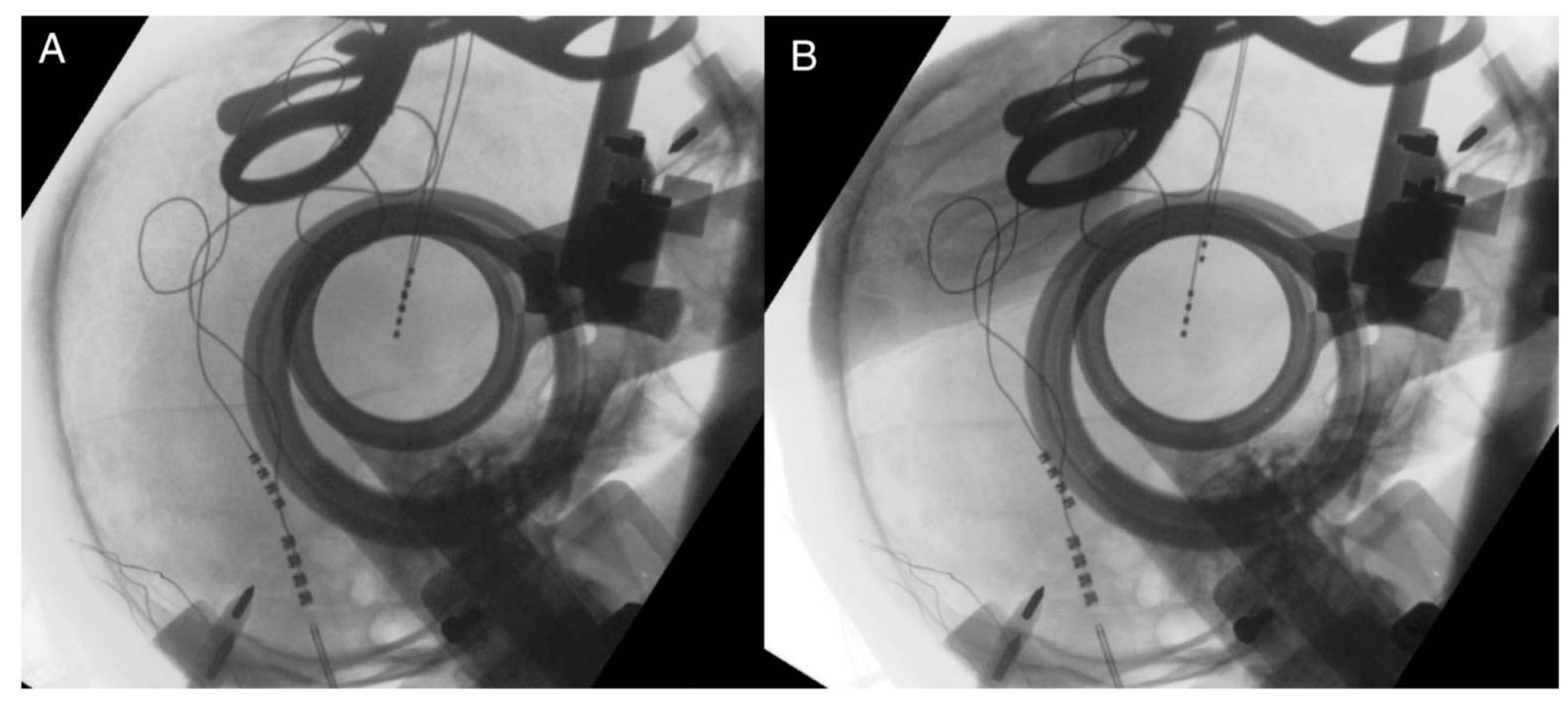

FIGURE 1.

Intraoperative lateral fluoroscopy showing deep brain stimulation leads (A) during the initial removal attempt and (B) after partial removal. 


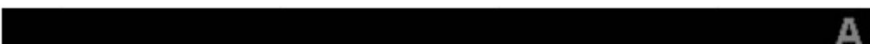

A

$\mathbf{R}$
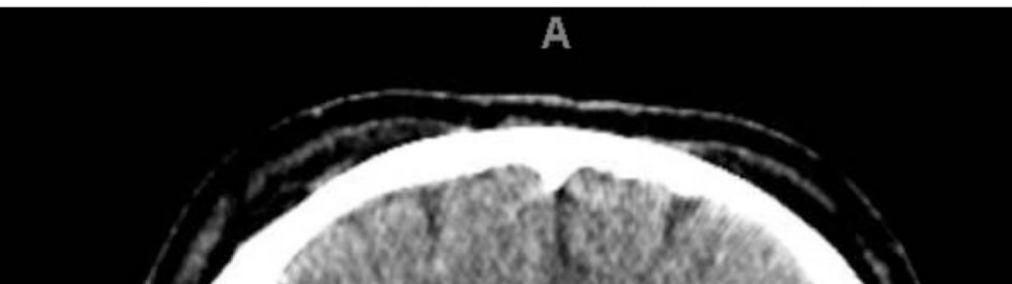

singy

atswowis

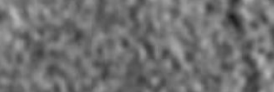




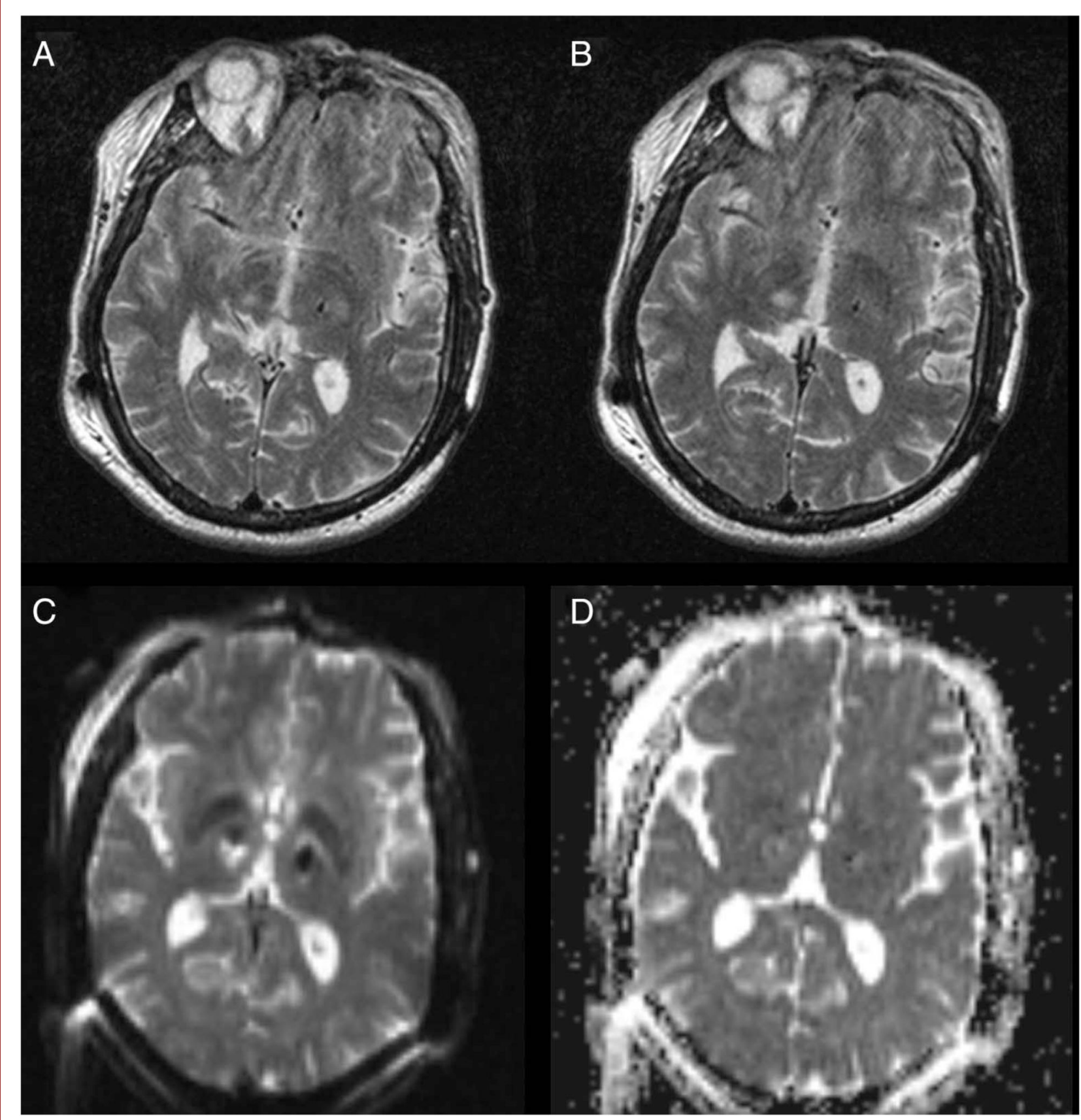

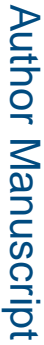

FIGURE 3.

Magnetic resonance images taken 24 hours postoperatively. A and B, T2-weighted images showing hyperintensity around the tip of the right lead. $\mathbf{C}$, diffusion-weighted imaging showing increased signal around the right lead tip. D, apparent diffusion coefficient map showing no corresponding diffusion restriction. 


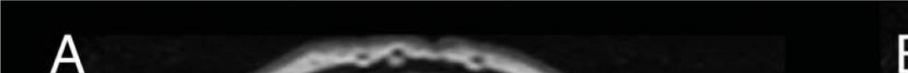

B
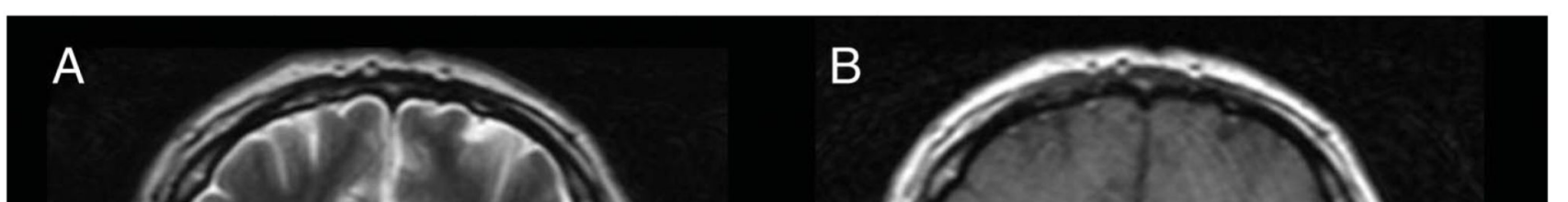

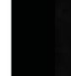

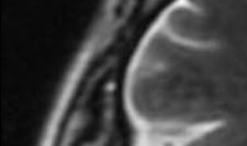

19) $=$

$-19$
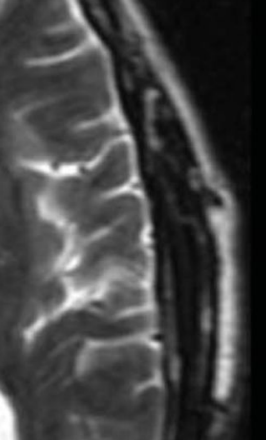

$\mathrm{e}^{4}$

$\int_{2}^{2}$

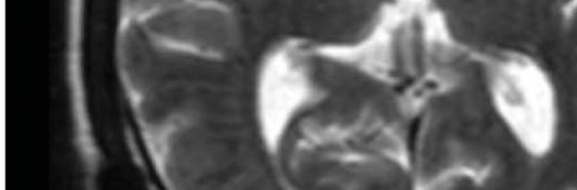

(.)

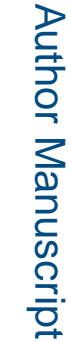

FIGURE 4.

Six-month follow-up magnetic resonance images. A, T2-weighted and (B) T1-weighted images showing a cystic space in the location of the partially removed right deep brain stimulation lead. 


\section{TABLE 1}

Stereotactic Coordinates of Previously Implanted Deep Brain Stimulation Leads ${ }^{a}$

\begin{tabular}{|lcr|}
\hline & \multicolumn{2}{c|}{ Lead Placement, $\mathbf{~ m m}$} \\
\cline { 2 - 3 } Coordinates & Left & Right \\
\hline AC-PC plane & & \\
\hline Lateral & -15.0 & 12.7 \\
\hline AP & -5.9 & -6.9 \\
\hline Vertical & 0.0 & 0.0 \\
\hline Anatomic & & \\
\hline $\begin{array}{l}\text { Distance lateral from the wall of } \\
\text { the third ventricle }\end{array}$ & 11.2 & 9.2 \\
\hline Distance anterior to the PC & 6.5 & 5.4 \\
\hline
\end{tabular}

${ }^{a}$ AC-PC, anterior commissure-posterior commissure; AP, anterior-posterior; PC posterior commissure. 

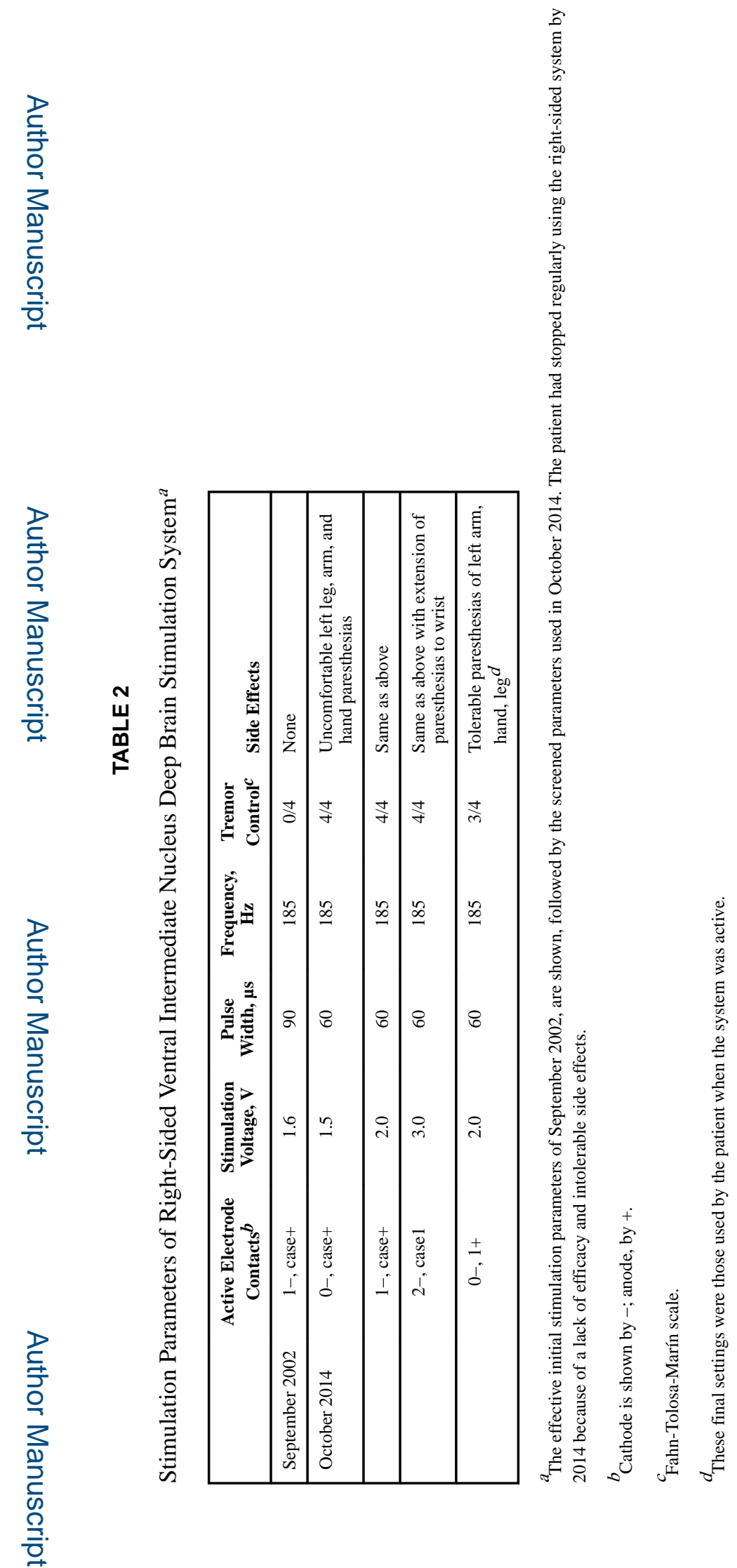

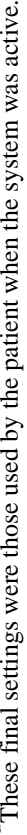

Neurosurgery. Author manuscript; available in PMC 2017 January 26. 